ISSN 0133-462X

hep-th/9609198
ITP Budapest Report No. 519

September 1996

\title{
On quantum equivalence of dual sigma models: $S L(3)$ examples
}

\author{
Z. Horváth, R.L. Karp and L. Palla \\ Institute for Theoretical Physics \\ Roland Eötvös University \\ H-1088 Budapest, Puskin u. 5-7, Hungary
}

\begin{abstract}
The equivalence of several $S L(3)$ sigma models and their special Abelian duals is investigated in the two loop order of perturbation theory. The investigation is based on extracting and comparing various $\beta$ functions of the original and dual models. The role of the discrete global symmetries is emphasized.
\end{abstract}




\section{Introduction}

Various target space duality transformations [1-3] ('T duality') connecting two seemingly different sigma-models or string-backgrounds are playing an increasingly important role nowadays, since they are assumed to lead to alternative descriptions of the same physical system. These transformations are the generalizations of the $R \rightarrow$ $1 / R$ duality in toroidal compactification of string theory, and are usually described in the sigma model formulation of the corresponding conformal field theory.

Using the sigma model formualtion it has been shown recently, both in the Abelian [4], and in the non Abelian case [5], that the duality transformation rules can be recovered in an elegant way by performing a canonical transformation. This clearly shows that the models related by these transformations are equivalent classically. In the quantum theory, the usual way to show that the models related by the duality transformations are equivalent, - in spite of the generally non linear change of variables they involve -, is by making some formal manipulations in the functional integral $[1,6]$, ignoring the need for regularization. While this may be sufficient for conformal invariant string backgrounds when no perturbative quantum corrections are expected, we feel, that from a pure $2 \mathrm{~d}$ field theory point of wiev, the question of quantum equivalence between sigma models related by duality transformations deserves further study.

We initiated such a study in ref.[7], where the various sigma models were treated as "ordinary" (i.e. not necessary conformally invariant) two dimensional quantum field theories in the framework of perturbation theory. Working in a field theoretic rather than string theoretic framework i.e. working with a flat, non dynamical 2 space and ignoring the dilaton, it was shown on a number of examples that the 'naive' (tree level) T-duality transformations in $2 \mathrm{~d} \sigma$-models cannot be exact symmetries of the quantum theory. The 'naive' Abelian duality transformations are correct to one loop in perturbation theory $\left({ }^{1}\right)$, they break down in general, however, at the two loop order. We reached these conclusions by analyzing and comparing various $\beta$ functions in the original and dual theories.

All the models investigated in ref.[7], - the deformed principal sigma model and its various duals -, were based on the group $S U(2)$. The aim of this paper is to check some of the ideas inferred from the study of these models in a slightly more complicated situation, i.e. when $S U(2)$ is replaced by $S L(3)$. In the very simple case of the Abelian duality transformations, when the distinguished component of the

$(1)$ see also ref.[8] 
original metric, $g_{00}$, (see eq.(3) below) is constant, the standard derivation using the functional integral amounts to just a standard gaussian integration, thus no problems are expected with the quantum equivalence of the dual theory. In ref.[7] it was found that in this case the dual model is indeed equivalent to two loops to the original one, however, there is a nontrivial change of scheme involved when insisting on dimensional regularization. We investigate below whether the same is true for the various deformed $S L(3)$ principal sigma models, when several, formally different dual models can be constructed with constant $g_{00}$. In this study we also clarify the role of the global symmetries - in particular the discrete ones.

The paper is organized as follows: in chapter 2 . we give a brief review of the duality transformations and the coupling constant renormalization procedure we use in the rest of the paper. In the first section of chapter 3. the Lagrangians of the various deformed $S L(3)$ principal models, admitting various degrees of discrete symmetries, are constructed, and in the second section of this chapter we derive the $\beta$ functions of the most symmetric of them. In chapter 4 . we study the renormalization of the two simplest duals of this model and show that the two loop counterterms contain new terms that cannot be explained by field redefinition thus naively these dual models are not renormalizable. However in the first section of chapter 5. we exhibit that working in an appropriately enlarged parameter space restores the renormalizability of the dual models and in the second section we indicate - using a generalized 'fixed point' analysis

- that even the physical $\beta$ functions of the original and dual models coincide, thereby confirming their equivalence. We summarize and make our conclusions in chapter 6 . The somewhat complicated expressions of the various two loop beta functions for the various models in the enlarged parameter space are collected in Appendix A, while the derivation of the two loop renorm invariants is sketched in Appendix B.

\section{Abelian $\mathbf{T}$ duality and coupling constant renormalization}

We start with a brief summary of the Abelian T-duality $[1,2,4]$. To this end consider the $\sigma$-model action:

$$
\begin{aligned}
S=\frac{1}{4 \pi \alpha^{\prime}} \int d^{2} z[ & \sqrt{h} h^{\mu \nu}\left(g_{00} \partial_{\mu} \theta \partial_{\nu} \theta+2 g_{0 \alpha} \partial_{\mu} \theta \partial_{\nu} \xi^{\alpha}+g_{\alpha \beta} \partial_{\mu} \xi^{\alpha} \partial_{\nu} \xi^{\beta}\right) \\
& \left.+\epsilon^{\mu \nu}\left(2 b_{0 \alpha} \partial_{\mu} \theta \partial_{\nu} \xi^{\alpha}+b_{\alpha \beta} \partial_{\mu} \xi^{\alpha} \partial_{\nu} \xi^{\beta}\right)\right]
\end{aligned}
$$

where $g_{i j}$ is the target space metric, $b_{i j}$ the torsion potential, and the target space indices are decomposed as $i=(0, \alpha)$ corresponding to the coordinate decomposition 
$\xi^{i}=\left(\theta, \xi^{\alpha}\right)$. The target space metric and $b_{i j}$ are assumed to possess a Killing vector and are now written in the adapted coordinate system, i.e. they are independent of the coordinate $\theta . h_{\mu \nu}$ is the world sheet metric and $\alpha^{\prime}$ the inverse of the string tension. The dilaton field is missing in eq.(1) as usual for non conformal sigma models: in this paper we are concerned mainly with asymptotically free models that are believed to generate non zero masses by dimensional transmutation when quantized as ordinary quantum field theories. In the same spirit the world sheet metric, $h_{\mu \nu}$, is taken to be flat in what follows. (e.g. that of a torus, to regulate the infrared divergences). Writing the integrand in eq.(1) as

$$
\mathcal{L}=g_{00}\left(\dot{\theta}^{2}-\theta^{\prime 2}\right)+\left(\dot{\theta}+\theta^{\prime}\right) J_{-}+\left(\dot{\theta}-\theta^{\prime}\right) J_{+}+V
$$

with

$$
\begin{aligned}
& J_{-}=\left(g_{0 i}+b_{0 i}\right) \partial_{-} \xi^{i}, \quad J_{+}=\left(g_{0 i}-b_{0 i}\right) \partial_{+} \xi^{i}, \\
& V=\left(g_{i j}+b_{i j}\right) \partial_{+} \xi^{i} \partial_{-} \xi^{j}, \quad z^{ \pm}=z^{0} \pm z^{1},
\end{aligned}
$$

we obtain the abelian dual with respect to the $\theta \rightarrow \theta+\alpha$ isometry by performing the canonical transformation [4]:

$$
p_{\theta}=-\tilde{\theta}^{\prime}, \quad p_{\tilde{\theta}}=-\theta^{\prime} .
$$

This transformation is mapping the 'original' $\sigma$-model with action, $S[g, b]$, given in eq. (1) to its dual, $S[\tilde{g}, \tilde{b}]$, where

$$
\begin{aligned}
& \tilde{g}_{00}=\frac{1}{g_{00}} \quad \tilde{g}_{0 \alpha}=\frac{b_{0 \alpha}}{g_{00}}, \quad \tilde{b}_{0 \alpha}=\frac{g_{0 \alpha}}{g_{00}} \\
& \tilde{g}_{\alpha \beta}=g_{\alpha \beta}-\frac{g_{0 \alpha} g_{0 \beta}-b_{0 \alpha} b_{0 \beta}}{g_{00}} \\
& \tilde{b}_{\alpha \beta}=b_{\alpha \beta}-\frac{g_{0 \alpha} b_{0 \beta}-g_{0 \beta} b_{0 \alpha}}{g_{00}} .
\end{aligned}
$$

The seemingly different models, described by $S[g, b]$ and $S[\tilde{g}, \tilde{b}]$, are classically equivalent as they are related by a canonical transformation. In principle, a possible way to investigate the equivalence of their quantized versions is by computing and comparing some 'physical' quantities up to an as high order of perturbative expansion as possible. (In practice, for sigma models with torsion, this is the two loop order). The perturbative determination of quantities like the free energy density or some scattering cross sections is greatly simplified if the model admits a sufficient degree of symmetry. Therefore we choose the original models to have enough symmetry to guarantee that 
they admit a parametrization, where their complete renormalization amounts to a multiplicative renormalization of the coupling constant(s) and the parameter(s); thus enabling the derivation of the corresponding $\beta$ functions $\left(^{2}\right)$. The derivation of these $\beta$ functions is the first step in the program of computing the aforementioned 'physical' quantities. Thus, as a first step, we inquire whether the $\beta$ functions, extracted from the dual models, are the same as the original ones.

Our general strategy to carry out the renormalization of the 'original' and of the 'dual' models and to obtain the corresponding $\beta$ functions is described in some details in ref.[7], thus here we just summarize what we need in the sequel. The procedure is based on the one resp. two loop counterterms for the general $\sigma$-models (either with or without the torsion term) computed by several authors [9-11]. These counterterms were derived by the background field method in the dimensional regularization scheme. Writing the general $\sigma$-model Lagrangian in the form

$$
\mathcal{L}=\frac{1}{2 \lambda}\left(g_{i j}(\xi)+b_{i j}(\xi)\right) \Xi^{i j}=\frac{1}{\lambda} \tilde{\mathcal{L}}, \quad \Xi^{i j}=\left(\partial_{\mu} \xi^{i} \partial^{\mu} \xi^{j}+\epsilon_{\mu \nu} \partial^{\mu} \xi^{i} \partial^{\nu} \xi^{j}\right)
$$

and expressing the loop expansion parameter, $\alpha^{\prime}$, in terms of the coupling constant, $\lambda$ as $\alpha^{\prime}=\lambda /(2 \pi)$, the simple pole parts of the one $(i=1)$ and two $(i=2)$ loop countertems, $\mathcal{L}_{i}$, apart from the $\mu^{-\epsilon}$ factor, are given as:

$$
\mu^{\epsilon} \mathcal{L}_{1}=\frac{\alpha^{\prime}}{2 \epsilon \lambda} \hat{R}_{i j} \Xi^{i j}=\frac{1}{\pi \epsilon} \Sigma_{1}
$$

and

$$
\mu^{\epsilon} \mathcal{L}_{2}=\frac{1}{2 \epsilon}\left(\frac{\alpha^{\prime}}{2}\right)^{2} \frac{1}{2 \lambda} Y_{j}^{l m k} \hat{R}_{i k l m} \Xi^{i j}=\frac{\lambda}{8 \pi^{2} \epsilon} \Sigma_{2}
$$

where

$$
\begin{aligned}
Y_{l m k j} & =-2 \hat{R}_{l m k j}+3 \hat{R}_{[k l m] j}+2\left(H^{2}\right)_{k l} g_{m j}-2\left(H^{2}\right)_{k m} g_{l j}, \\
\left(H^{2}\right)_{i j} & =H_{i k l} H_{j}^{k l}, \quad 2 H_{i j k}=\partial_{i} b_{j k}+\text { cyclic, }
\end{aligned}
$$

In these equations $\hat{R}_{i k l m}$ resp. $\hat{R}_{i j}$ denote the generalized Riemann resp. Ricci tensors of the generalized connection, $G_{j k}^{i}$, containing also the torsion term in addition to the Christoffel symbols $\Gamma_{j k}^{i}$, of the metric $g_{i j}: G_{j k}^{i}=\Gamma_{j k}^{i}+H_{j k}^{i}$.

If the metric, $g_{i j}$, and the torsion potential, $b_{i j}$, depend also on a parameter, $x$; $g_{i j}=g_{i j}(\xi, x), b_{i j}=b_{i j}(\xi, x)$ then we convert the previous counterterms into coupling

$\left({ }^{2}\right)$ The difference between a coupling constant and a parameter is that the parameter is not necessarily small, thus we do not expand anything in the parameter, while in the coupling constant we assume a perturbative expansion. 
constant and parameter renormalization by assuming that in the one $(i=1)$ and two $(i=2)$ loop orders their bare and renormalized values are related as

$$
\begin{aligned}
& \lambda_{0}=\mu^{\epsilon} \lambda\left(1+\frac{\zeta_{1}(x) \lambda}{\pi \epsilon}+\frac{\zeta_{2}(x) \lambda^{2}}{8 \pi^{2} \epsilon}+\ldots\right)=\mu^{\epsilon} \lambda Z_{\lambda}(x, \lambda)=\mu^{\epsilon} \lambda\left(1+\frac{y_{\lambda}(\lambda, x)}{\epsilon}+\ldots\right), \\
& x_{0}=x+\frac{x_{1}(x) \lambda}{\pi \epsilon}+\frac{x_{2}(x) \lambda^{2}}{8 \pi^{2} \epsilon}+\ldots=x Z_{x}(x, \lambda)=x\left(1+\frac{y_{x}(\lambda, x)}{\epsilon}+\ldots\right),
\end{aligned}
$$

(where the ellipses stand for both the higher loop contributions and for the higher order pole terms). We determine the unknown functions, $\zeta_{i}(x)$ and $x_{i}(x),(i=1,2)$, from the equations:

$$
-\zeta_{i}(x) \tilde{\mathcal{L}}+\frac{\partial \tilde{\mathcal{L}}}{\partial x} x_{i}(x)+\frac{\delta \tilde{\mathcal{L}}}{\delta \xi^{k}} \xi_{i}^{k}(\xi, x)=\Sigma_{i}, \quad i=1,2
$$

These equations express the finiteness of the generalized quantum effective action, $\Gamma(\xi)$, as defined in ref.s[12], up to the corresponding orders in perturbation theory. In eq. (9) $\xi_{i}^{k}(\xi, x)$ may depend in an arbitrary way on the parameter, $x$, and on the fields, $\xi^{j}$, the only requirement is that $\xi_{i}^{k}(\xi, x)$ may contain no derivatives of $\xi^{j}$. Eq.(9) admits a simple interpretation: it suggests that the general counterterms of the sigma models may be accounted for by the coupling constant and parameter renormalization if the latter ones are accompanied by a (in general non-linear) redefinition of the fields $\xi^{j}$ :

$$
\xi_{0}^{j}=\xi^{j}+\frac{\xi_{1}^{j}\left(\xi^{k}, x\right) \lambda}{\pi \epsilon}+\frac{\xi_{2}^{j}\left(\xi^{k}, x\right) \lambda^{2}}{8 \pi^{2} \epsilon}+\ldots
$$

in such a way that eq.(9) holds $\left({ }^{3}\right)$. In the special case when $\xi_{i}^{k}$ depends linearly on the $\xi$ fields, $\xi_{i}^{k}(\xi, x)=\xi^{k} y_{i}^{k}(x)$, eq.(10) simplifies to an ordinary multiplicative wave function renormalization. We emphasize that it is not guaranteed a priori that eq.(9) may be solved for $\zeta_{i}(x), x_{i}(x)$ and $\xi_{i}^{k}(\xi, x)$. If $\Sigma_{i}$ happen to have a form that makes this impossible then this implies, that the renormalization of the model drives it - in the infinite dimensional space of all metrics and torsion potentials out of the lower dimensional subspace characterized by the coupling constant(s) and the parameter(s); i.e. implies that the model is not renormalizable in the ordinary, field theoretical sense. On the other hand, if eq.(9) admits a solution, then, writing $Z_{\lambda}=1+\frac{y_{\lambda}(\lambda, x)}{\epsilon}+\ldots$ and $Z_{x}=1+\frac{y_{x}(\lambda, x)}{\epsilon}+\ldots$, the $\beta$ functions of $\lambda$ and $x$, defined

$\left({ }^{3}\right)$ Note that here $\xi^{j}$ is not assumed to solve any equation of motion so it is not related directly to the 'classical' field of ref.s[12] 
in the standard way, are readily obtained:

$$
\beta_{\lambda}=\lambda^{2} \frac{\partial y_{\lambda}}{\partial \lambda}, \quad \beta_{x}=x \lambda \frac{\partial y_{x}}{\partial \lambda}
$$

This framework was used in [7] in several examples of $S U(2)$-based sigma models to investigate whether the same $\beta$ functions can be extracted from the original and the dual models.

\section{3. 'Deformed' $S L(3)$ principal sigma models}

\subsection{General considerations}

Below we consider various versions of the deformed $S L(3)$ principal sigma model and its abelian duals, although models built from the elements of $S U(3)$ were the most natural generalizations of the examples investigated in ref.[7]. The reason for this is twofold: on the one hand working with the non compact version makes it possible to use the Gauss decomposition to parametrize the group elements, which yields, eventually, an action polynomial (rather than trigonometric) in the fields; while, on the other, we know [13] that the compact and non compact versions are on the same footing as far as the beta functions are concerned. Therefore we parametrize $G \in S L(3)$ by a lower triangular, an upper triangular and a diagonal matrix as

$$
G=G_{U} G_{L} G_{D}
$$

where

$$
\begin{gathered}
G_{U}=\left(\begin{array}{ccc}
1 & \mathrm{~F}_{6}(x, t) & \mathrm{F}_{8}(x, t) \\
0 & 1 & \mathrm{~F}_{7}(x, t) \\
0 & 0 & 1
\end{array}\right), \quad G_{L}=\left(\begin{array}{ccc}
1 & 0 & 0 \\
\mathrm{~F}_{3}(x, t) & 1 & 0 \\
\mathrm{~F}_{5}(x, t) & \mathrm{F}_{4}(x, t) & 1
\end{array}\right), \\
G_{D}=\left(\begin{array}{ccc}
e^{\mathrm{F}_{1}(x, t)+\frac{\mathrm{F}_{2}(x, t)}{\sqrt{3}}} & 0 \\
0 & e^{-\mathrm{F}_{1}(x, t)+\frac{\mathrm{F}_{2}(x, t)}{\sqrt{3}}} & 0 \\
0 & 0 & 0 \\
0 & e^{-\frac{2 \mathrm{~F}_{2}(x, t)}{\sqrt{3}}}
\end{array}\right),
\end{gathered}
$$

and use $\mathrm{F}_{i}(x, t)(i=1, \ldots, 8)$ as the fields $\xi_{i}$.

To construct the 'original' model we follow the philosophy of ref.[7] in choosing it as symmetric as possible, since in this way we can keep the number of parameters 
that require renormalization small. Therefore we build the models we start with from the various components of the current, $J_{\mu}=G^{-1} \partial_{\mu} G=J_{\mu}^{a} \lambda^{a}$ :

$$
J_{\mu}=\left(\begin{array}{ccc}
J_{\mu}^{3}+\frac{1}{\sqrt{3}} J_{\mu}^{8} & J_{\mu}^{1} & J_{\mu}^{4} \\
J_{\mu}^{2} & -J_{\mu}^{3}+\frac{1}{\sqrt{3}} J_{\mu}^{8} & J_{\mu}^{6} \\
J_{\mu}^{5} & J_{\mu}^{7} & -\frac{2}{\sqrt{3}} J_{\mu}^{8}
\end{array}\right)
$$

since this way invariance under the 'left' $S L(3)$ transformations, generated by constant $G_{0}$ elements of $S L(3)$, multiplying $G$, eq.(12), from the left, $G \rightarrow G_{0} G$, is automatically guaranteed. The Lagrangians of the various 'deformed' principal models are obtained by adding various quadratic expressions, $J_{\mu}^{a} J^{\mu b}$, to the Lagrangian of the principal model:

$$
\mathcal{L}_{\mathrm{pr}}=-\frac{1}{2 \lambda} \operatorname{Tr} J_{\mu} J^{\mu}
$$

so as to break its invariance under the 'right' $S L(3)$ transformations $\left(G \rightarrow G G_{0}\right)$ to an appropriate subgroup. We certainly want this unbroken right invariance group to contain $\mathbf{R} \times \mathbf{R}=\mathbf{R}^{2}$, formed by the $\mathrm{F}_{1} \rightarrow \mathrm{F}_{1}+\alpha$ and $\mathrm{F}_{2} \rightarrow \mathrm{F}_{2}+\beta$ translations of $\mathrm{F}_{1}$ and $\mathrm{F}_{2}$, since we need these invariances to construct the various duals. As these translations are generated by

$$
\left(\begin{array}{ccc}
e^{\alpha} & 0 & 0 \\
0 & e^{-\alpha} & 0 \\
0 & 0 & 1
\end{array}\right) \quad \text { and } \quad\left(\begin{array}{ccc}
e^{\frac{\beta}{\sqrt{3}}} & 0 & 0 \\
0 & e^{\frac{\beta}{\sqrt{3}}} & 0 \\
0 & 0 & e^{\frac{-2 \beta}{\sqrt{3}}}
\end{array}\right)
$$

an easy calcualtion shows that the following $\mathbf{R}^{2}$ invariant combinations are possible: $\left(J_{\mu}^{3}\right)^{2},\left(J_{\mu}^{8}\right)^{2}, J_{\mu}^{1} J^{2 \mu}, J_{\mu}^{4} J^{\mu 5}, J_{\mu}^{6} J^{\mu 7}$. Therefore, an arbitrary linear combination of these terms could be added to eq.(16) to obtain an $S L(3)_{L} \times \mathbf{R}_{R}^{2}$ symmetric deformed principal sigma model. Note, however, that after an appropriate rescaling of $\lambda$ in eq.(16) and the coefficients of the linear combination, any one of the previous invariants can be absorbed into the term describing the principal model. Thus the maximum number of free parameters characterizing the sigma models with $S L(3)_{L} \times \mathbf{R}_{R}^{2}$ symmetry in addition to the coupling, $\lambda$, is four. To reduce this number we look for discrete subgroups of $S L(3)$ that can be imposed as symmetries in addition to $\mathbf{R}^{2}$. The most natural candidates are the $\mathbf{Z}_{2}$ subgroups generated by the elements:

$$
M_{1}=\left(\begin{array}{lll}
0 & 1 & 0 \\
1 & 0 & 0 \\
0 & 0 & 1
\end{array}\right), \quad M_{2}=\left(\begin{array}{ccc}
1 & 0 & 0 \\
0 & 0 & 1 \\
0 & 1 & 0
\end{array}\right), \quad M_{3}=\left(\begin{array}{lll}
0 & 0 & 1 \\
0 & 1 & 0 \\
1 & 0 & 0
\end{array}\right)
$$


and the $\mathbf{Z}_{3}$ subgroup generated by

$$
z_{1}=\left(\begin{array}{ccc}
0 & 1 & 0 \\
0 & 0 & 1 \\
1 & 0 & 0
\end{array}\right), \quad z_{1}^{2}=z_{2}=\left(\begin{array}{ccc}
0 & 0 & 1 \\
1 & 0 & 0 \\
0 & 1 & 0
\end{array}\right) .
$$

A short calculation, using the action of these elements on $G\left(G \rightarrow a^{-1} G a ; a=M_{i}\right.$, $z, z^{2}$ ) and the definition of the current, shows that

$$
\alpha\left(J_{\mu}^{3}\right)^{2}+\beta\left(J_{\mu}^{8}\right)^{2}+\delta J_{\mu}^{1} J^{2 \mu}+\gamma\left(J_{\mu}^{4} J^{\mu 5}+J_{\mu}^{6} J^{\mu 7}\right)
$$

is invariant under $M_{1}$,

$$
\alpha\left(\left(J_{\mu}^{3}\right)^{2}+\left(J_{\mu}^{8}\right)^{2}\right)+\delta J_{\mu}^{6} J^{7 \mu}+\gamma\left(J_{\mu}^{4} J^{\mu 5}+J_{\mu}^{1} J^{\mu 2}\right)
$$

is invariant under $M_{2}$,

$$
\alpha\left(\left(J_{\mu}^{3}\right)^{2}+\left(J_{\mu}^{8}\right)^{2}\right)+\delta J_{\mu}^{4} J^{5 \mu}+\gamma\left(J_{\mu}^{1} J^{\mu 2}+J_{\mu}^{6} J^{\mu 7}\right)
$$

is invariant under $M_{3}$, and

$$
\alpha\left(\left(J_{\mu}^{3}\right)^{2}+\left(J_{\mu}^{8}\right)^{2}\right)+\beta\left(J_{\mu}^{4} J^{5 \mu}+J_{\mu}^{1} J^{\mu 2}+J_{\mu}^{6} J^{\mu 7}\right)
$$

is invariant under $\mathbf{Z}_{3}$. Note that requiring the $\mathbf{Z}_{3}$ symmetry implies the various $\mathbf{Z}_{2}$ symmetries as well, i.e. choosing eq.(22) guarantees that the cyclic $\mathbf{Z}_{3}$ symmetry is enhanced to $\mathrm{S}_{3}$. We mention in passing that although the action of these discrete subgroups on $J_{\mu}^{a}$ is simple, on the fields, $\mathrm{F}_{i}$, they act in general in a rather non-trivial way; e.g. under $M_{1}$ the various $\mathrm{F}_{i}$ 's transform as

$$
\begin{aligned}
& \mathrm{F}_{1} \rightarrow-\mathrm{F}_{1}-\ln \left(1+\mathrm{F}_{3} \mathrm{~F}_{6}\right), \quad \mathrm{F}_{2} \rightarrow \mathrm{F}_{2}, \\
& \mathrm{~F}_{7} \rightarrow \mathrm{F}_{8}, \quad \mathrm{~F}_{8} \rightarrow \mathrm{F}_{7}, \quad \mathrm{~F}_{4} \rightarrow \frac{\mathrm{F}_{5}}{1+\mathrm{F}_{3} \mathrm{~F}_{6}}, \\
& \mathrm{~F}_{6} \rightarrow \frac{\mathrm{F}_{3}}{1+\mathrm{F}_{3} \mathrm{~F}_{6}}, \quad \mathrm{~F}_{5} \rightarrow \mathrm{F}_{4}\left(1+\mathrm{F}_{3} \mathrm{~F}_{6}\right), \quad \mathrm{F}_{3} \rightarrow \mathrm{F}_{6}\left(1+\mathrm{F}_{3} \mathrm{~F}_{6}\right) .
\end{aligned}
$$

\subsection{The simplest deformed principal model and its renormalization}

Using the aforementioned rescaling argument to absorb the second term in eq.(22) into the expression of the principal model we write the Lagrangian of the 'most symmetric' deformed $S L(3)$ principal model - that contain just one parameter in addition to $\lambda$ - as:

$$
\mathcal{L}=-\frac{1}{2 \lambda}\left(\operatorname{Tr} J_{\mu} J^{\mu}+2 g\left(\left(J_{\mu}^{3}\right)^{2}+\left(J_{\mu}^{8}\right)^{2}\right)\right)=\frac{1}{2 \lambda} g_{i j}(\mathrm{~F}, g) \partial^{\mu} \mathrm{F}^{i} \partial_{\mu} \mathrm{F}^{j} .
$$


Here, by writing the second equality, we emphasize that this model is a purely metric one, with $g_{i j}$ depending also on the 'deformation' parameter $g$. Since the translations of $\mathrm{F}_{1}$ and $\mathrm{F}_{2}$ do not commute with the action of $\mathrm{S}_{3}$, the total global symmetry of this Lagrangian is the semidirect product: $S L(3)_{L} \times \mathbf{R}_{R}^{2} \bowtie_{s} S_{3}$.

Explicitely, the non vanishing components of the metric are:

$$
\begin{aligned}
& g_{11}=-2-2 g \quad g_{16}=-2(1+g) \mathrm{F}_{3} \quad g_{17}=(1+g)\left(\mathrm{F}_{4} \mathrm{~F}_{6} \mathrm{~F}_{3}+\mathrm{F}_{4}+\mathrm{F}_{6} \mathrm{~F}_{5}\right) \\
& g_{18}=-(1+g)\left(\mathrm{F}_{3} \mathrm{~F}_{4}+\mathrm{F}_{5}\right) \quad g_{22}=-2-2 g \\
& g_{27}=-\sqrt{3}\left(\mathrm{~F}_{4} \mathrm{~F}_{6} \mathrm{~F}_{3}-\mathrm{F}_{6} \mathrm{~F}_{5}+\mathrm{F}_{4}\right)(1+g) \\
& g_{28}=\sqrt{3}\left(\mathrm{~F}_{3} \mathrm{~F}_{4}-\mathrm{F}_{5}\right)(1+g) \quad g_{36}=-1 \quad g_{47}=-1-\mathrm{F}_{6} \mathrm{~F}_{3} \quad g_{48}=\mathrm{F}_{3} \quad g_{57}=\mathrm{F}_{6} \\
& g_{66}=-2 g \mathrm{~F}_{3}^{2} g_{67}=g \mathrm{~F}_{3}\left(\mathrm{~F}_{4} \mathrm{~F}_{6} \mathrm{~F}_{3}+\mathrm{F}_{4}+\mathrm{F}_{6} \mathrm{~F}_{5}\right) \quad g_{68}=-g \mathrm{~F}_{3}\left(\mathrm{~F}_{3} \mathrm{~F}_{4}+\mathrm{F}_{5}\right) \\
& g_{77}=-2 g\left(-\mathrm{F}_{5} \mathrm{~F}_{3} \mathrm{~F}_{4} \mathrm{~F}_{6}^{2}+\mathrm{F}_{4}^{2}+2 \mathrm{~F}_{3} \mathrm{~F}_{4}^{2} \mathrm{~F}_{6}+\mathrm{F}_{3}^{2} \mathrm{~F}_{4}^{2} \mathrm{~F}_{6}^{2}+\mathrm{F}_{5}^{2} \mathrm{~F}_{6}^{2}-\mathrm{F}_{5} \mathrm{~F}_{4} \mathrm{~F}_{6}\right) \\
& g_{78}=g\left(2 \mathrm{~F}_{3}^{2} \mathrm{~F}_{4}^{2} \mathrm{~F}_{6}+2 \mathrm{~F}_{5}^{2} \mathrm{~F}_{6}-2 \mathrm{~F}_{5} \mathrm{~F}_{3} \mathrm{~F}_{4} \mathrm{~F}_{6}-\mathrm{F}_{5} \mathrm{~F}_{4}+2 \mathrm{~F}_{3} \mathrm{~F}_{4}^{2}\right) \\
& g_{85}=-1 g_{88}=-2 g\left(\mathrm{~F}_{3}^{2} \mathrm{~F}_{4}^{2}+\mathrm{F}_{5}^{2}-\mathrm{F}_{3} \mathrm{~F}_{5} \mathrm{~F}_{4}\right) .
\end{aligned}
$$

Now we discuss briefly the implementation of the coupling constant renormalization procedure for this model.

Computing the counterterms - using eq.(5-7) - for the Lagrangian in eq.(24) reveals that both $\Sigma_{1}$ and $\Sigma_{2}$ preserve the structure of the metric, eq.(25): all the non vanishing terms of $\Sigma_{1}$ and $\Sigma_{2}$ have the same polynomial forms in $\mathrm{F}_{i}$ and $\partial_{\mu} \mathrm{F}_{i}$ as in eq. (24), the only change is in the coefficients of the various terms. Therefore we try to abstract the renormalization of $\lambda$ and $g$ by assuming an ordinary wave function renormalization for the $\mathrm{F}_{i}$ fields, i.e. we substitute

$$
F_{i}^{0}=Z_{i}(g, \lambda) F_{i} \quad i=\overline{1,8}, \quad g_{0}=Z_{g}(g, \lambda) g, \quad \lambda^{0}=Z_{\lambda}(g, \lambda) \lambda
$$

with

$$
\begin{aligned}
& Z_{i}(g, \lambda)=1+\frac{1}{\epsilon}\left(\frac{y_{i}^{(1)}(g) \lambda}{\pi}+\frac{y_{i}^{(2)}(g) \lambda^{2}}{8 \pi^{2}}+\ldots\right)+\ldots \\
& Z_{g}(g, \lambda)=1+\frac{y_{g}(g, \lambda)}{\epsilon}+\ldots, \quad Z_{\lambda}(g, \lambda)=1+\frac{y_{\lambda}(g, \lambda)}{\epsilon}+\ldots,
\end{aligned}
$$

into eq. $(9,10)$ in both the one and two loop order. (In eq.(27) $y_{\lambda}$ and $y_{g}$ are given by eq.(8), after the obvious $\zeta_{i}(x) \rightarrow y_{\lambda}^{i}(g)$ and $x_{i}(x) \rightarrow y_{g}^{i}(g) i=1,2$ changes). Eq.(9) yields, in both cases, 66 linear equations for the 10 unkowns $y_{i}^{(j)}(g), i=\overline{1,8}, y_{\lambda}^{j}(g)$, $y_{g}^{j}(g)$; however as a result of the high degree of symmetry of eq.(24) and the manifest target space covariance of the background field method this system is solvable. In 
fact $y_{\lambda}^{j}(g)$ and $y_{g}^{j}(g)$ are determined uniquely, while there is a two parameter freedom in the choice of the 8 wave function renormalization constants $y_{i}^{(j)}(g)$. This freedom reflects the following two independent scaling invariances of the Lagrangian, eq.(24):

$$
\begin{aligned}
& F_{1} \longrightarrow\left(\begin{array}{c}
1 \\
1
\end{array}\right) F_{1} \quad F_{2} \longrightarrow\left(\begin{array}{l}
1 \\
1
\end{array}\right) F_{2} \quad F_{3} \longrightarrow\left(\begin{array}{c}
e^{\gamma} \\
e^{\delta}
\end{array}\right) F_{3} \quad F_{4} \longrightarrow\left(\begin{array}{c}
e^{-\gamma} \\
1
\end{array}\right) F_{4} \\
& F_{5} \longrightarrow\left(\begin{array}{c}
1 \\
e^{\delta}
\end{array}\right) F_{5} \quad F_{6} \longrightarrow\left(\begin{array}{c}
e^{-\gamma} \\
e^{-\delta}
\end{array}\right) F_{6} \quad F_{7} \longrightarrow\left(\begin{array}{c}
e^{\gamma} \\
1
\end{array}\right) F_{7} \quad F_{8} \longrightarrow\left(\begin{array}{c}
1 \\
e^{-\delta}
\end{array}\right) F_{8} .
\end{aligned}
$$

In one loop the explicit form of the coupling constant and deformation parameter's renormalization constants are:

$$
y_{g}(g, \lambda)=\frac{5 \lambda(1+g)}{4 \pi}, \quad y_{\lambda}(g, \lambda)=\frac{(2 g-3) \lambda}{4 \pi},
$$

while in two loops:

$$
\begin{aligned}
& y_{g}(g, \lambda)=-\frac{\lambda(1+g)(10 g \lambda-13 \lambda-40 \pi)}{32 \pi^{2}} \\
& y_{\lambda}(g, \lambda)=-\frac{\lambda\left(48 \pi+26 \lambda g^{2}-32 \pi g-11 g \lambda+9 \lambda\right)}{64 \pi^{2}},
\end{aligned}
$$

was obtained. Using these expressions in eq.(11) leads to the following one and two loop $\beta$ functions:

$$
\begin{gathered}
\beta_{\lambda}^{(1)}=\frac{\lambda^{2}(2 g-3)}{4 \pi}, \\
\beta_{g}^{(1)}=\frac{5 \lambda(1+g) g}{4 \pi}, \\
\beta_{\lambda}^{(2)}=\frac{\lambda^{2}(2 g-3)}{4 \pi}+\frac{\lambda^{3}\left(26 g^{2}-11 g+9\right)}{32 \pi^{2}}, \\
\beta_{g}^{(2)}=\frac{5 \lambda(1+g) g}{4 \pi}+\frac{\lambda^{2} g\left(10 g^{2}-3 g-13\right)}{16 \pi^{2}} .
\end{gathered}
$$

It is easy to see that the $g=0$ resp. the $g=-1$ lines are fixed lines under the renormalization group in the $(\lambda, g)$ plane. On the $g=0$ line (that corresponds to the principal model) $\beta_{\lambda}^{(2)}(\lambda)$ indeed reduces to the well known $\beta$ function of the $S U(3)$ principal model [14] if we take into account the normalization of $\lambda$ implied by eq.(16). Looking at eq.s $(24,25)$ we conclude that the $g=-1$ fixed line - that also describes an asymptotically free model - can be interpreted as a sort of "coset model" where the $J_{\mu}^{3}, J_{\mu}^{8}$ current components - or the $\mathrm{F}_{1}$ and $\mathrm{F}_{2}$ fields, decouple completely. Away from these lines in the $(\lambda \geq 0, g<0)$ quarter of the $(\lambda, g)$ plane the renorm trajectories run 
into $\lambda=0, g=-1$; while for $g>0$ they run to infinity. This implies that the $g=0$ fixed line corresponding to the principal $\sigma$-model is 'unstable' under the deformation.

As the metric, eq.(25), is independent of $\mathrm{F}_{1}, \mathrm{~F}_{2}, \mathrm{~F}_{7}$ and $\mathrm{F}_{8}$, the simplest deformed $S L(3)$ principal model has four Abelian isometries (corresponding to the translations of these fields), that can be used to construct various dual models. Of these four possibilities we are concerned here only with the ones using the $\mathrm{F}_{1}$ and $\mathrm{F}_{2}$ translations as $g_{11}$ and $g_{22}$ are constants, while $g_{77}$ and $g_{88}$ depend on the other F's. In the sequel we call ' $\mathrm{F}_{1}$ (resp. $\mathrm{F}_{2}$ ) dual' of any 'original' model the dual models obtained by using the $\mathrm{F}_{1}$ (resp. $\mathrm{F}_{2}$ ) translations to generate them.

\section{The $\mathrm{F}_{1}$ dual of the simplest deformed principal model}

The Lagrangian of the $\mathrm{F}_{1}$ dual of the simplest deformed principal model, $\mathcal{L}^{d}$, is easily obtained from using eq.(3) and eq.(24-25). Interestingly this dual model contains a non trivial torsion term, in addition to a somewhat different metric. Appropriately rescaling the field dual to $\mathrm{F}_{1}$ and - with a slight abuse of notation - still denoting it by $\mathrm{F}_{1}$, the non vanishing components of $b_{i j}$ and $g_{i j}$ are:

$$
\begin{aligned}
& b_{16}=\sqrt{1+g} \mathrm{~F}_{3}, \quad b_{17}=-\frac{1}{2} \sqrt{1+g}\left(\mathrm{~F}_{4} \mathrm{~F}_{6} \mathrm{~F}_{3}+\mathrm{F}_{4}+\mathrm{F}_{6} \mathrm{~F}_{5}\right) \\
& b_{18}=\frac{1}{2} \sqrt{1+g}\left(\mathrm{~F}_{3} \mathrm{~F}_{4}+\mathrm{F}_{5}\right) \\
& g_{11}=-\frac{1}{2} g_{22}=-2-2 g \quad g_{27}=-\sqrt{3}\left(\mathrm{~F}_{4} \mathrm{~F}_{6} \mathrm{~F}_{3}-\mathrm{F}_{6} \mathrm{~F}_{5}+\mathrm{F}_{4}\right)(1+g) \\
& g_{28}=\sqrt{3}\left(\mathrm{~F}_{3} \mathrm{~F}_{4}-\mathrm{F}_{5}\right)(1+g) \quad g_{36}=-1 g_{47}=-1-\mathrm{F}_{6} \mathrm{~F}_{3} \quad g_{48}=\mathrm{F}_{3} \quad g_{57}=\mathrm{F}_{6} \\
& g_{66}=2 \mathrm{~F}_{3}^{2} g_{67}=-\mathrm{F}_{3}\left(\mathrm{~F}_{4} \mathrm{~F}_{6} \mathrm{~F}_{3}+\mathrm{F}_{4}+\mathrm{F}_{6} \mathrm{~F}_{5}\right) \quad g_{68}=\mathrm{F}_{3}\left(\mathrm{~F}_{3} \mathrm{~F}_{4}+\mathrm{F}_{5}\right) \\
& g_{77}=(3 g+1) \mathrm{F}_{5} \mathrm{~F}_{4} \mathrm{~F}_{6}\left(\mathrm{~F}_{6} \mathrm{~F}_{3}+1\right)+ \\
& \frac{1-3 g}{2}\left(\mathrm{~F}_{4}^{2}+2 \mathrm{~F}_{3} \mathrm{~F}_{4}{ }^{2} \mathrm{~F}_{6}+\mathrm{F}_{3}{ }^{2} \mathrm{~F}_{4}{ }^{2} \mathrm{~F}_{6}{ }^{2}+\mathrm{F}_{5}{ }^{2} \mathrm{~F}_{6}{ }^{2}\right) \\
& g_{78}=\frac{1-3 g}{2}\left(2 \mathrm{~F}_{3} \mathrm{~F}_{4}{ }^{2} \mathrm{~F}_{6}+\mathrm{F}_{3}{ }^{2} \mathrm{~F}_{4}{ }^{2} \mathrm{~F}_{6}+\mathrm{F}_{5}{ }^{2} \mathrm{~F}_{6}{ }^{2}+\mathrm{F}_{4}{ }^{2}\right) \\
& +(3 g+1) \mathrm{F}_{5} \mathrm{~F}_{4} \mathrm{~F}_{6}\left(\mathrm{~F}_{3} \mathrm{~F}_{6}+{ }_{1}\right) \\
& g_{85}=-1 \quad g_{88}=\frac{1}{2}(1-3 g)\left(\mathrm{F}_{3}{ }^{2} \mathrm{~F}_{4}{ }^{2}+\mathrm{F}_{5}{ }^{2}\right)+(3 g+1) \mathrm{F}_{3} \mathrm{~F}_{5} \mathrm{~F}_{4}
\end{aligned}
$$

Note that the global symmetries of this dual model consist of the full $S L(3)_{L}$ transformations (that include among others the translations of $F_{7}$ and $F_{8}$ as well as the scaling transformations of eq.(28)) and the translations of $\mathrm{F}_{1}$ and $\mathrm{F}_{2}$. The discrete $\mathrm{S}_{3}$ 
part of the original symmetry group is missing: the $\mathrm{F}_{1}$ translation used to construct the dual does not commute with it.

Applying the coupling constant renormalization procedure in the same way as for the purely metric 'original' model leads, in the one loop order, to the same $\beta$ functions, eq.(31). However for two loops the situation changes dramatically: the structure of the Lagrangian is not reproduced by the countertems as completely new terms appear in the symmetric part of $\Sigma_{2}$ :

$$
\alpha_{0}\left(\mathrm{~F}_{4} \mathrm{~F}_{6} \partial_{\mu} \mathrm{F}_{3} \partial^{\mu} \mathrm{F}_{7}-\mathrm{F}_{4} \partial_{\mu} \mathrm{F}_{3} \partial^{\mu} \mathrm{F}_{8}+\mathrm{F}_{5} \partial_{\mu} \mathrm{F}_{6} \partial^{\mu} \mathrm{F}_{7}\right)
$$

where $\alpha_{0}=3 \lambda^{2}(1+g)(5-3 g) /\left(32 \pi^{2}\right)$. These terms are new, since in the metric, eq. $(34), g_{37}$ and $g_{38}$ vanish and $g_{67}$ contains no single $\mathrm{F}_{5}$. We note, that these new terms are manifestly invariant under the four translational and two scaling (eq.(28)) invariances of $\mathcal{L}^{d}$, a fact reflecting that dimensional regularization preserves the global symmetries.

To establish the two loop equivalence of this dual model to eq.(24) we must be able, as a first step, to extract from $\Sigma_{2}$ the two loop renormalization constants of $\lambda$ and $g$. If this is completed, then we have to inquire whether the $\beta$-functions obtained from these renormalization constants are really equivalent - possibly after an appropriate change of the scheme [7] - to those in eq.(32).

To extract the renormalization constants of $\lambda$ and $g$ we look for reparametrizations of $\mathrm{F}_{\mathrm{i}}$ 's that would account for the new terms in eq.(35) while preserving the manifest symmetries (the four translations and the two scaling invariances in eq.(28)). It is easy to see, that

$$
\mathrm{F}_{5} \rightarrow \mathrm{F}_{5}+\alpha_{0} \mathrm{~F}_{3} \mathrm{~F}_{4} / \epsilon
$$

accounts for the the first two terms in eq.(35) while it preserves the polynomial form of the other metric and torsion components in eq. $(33,34)$. However we proved that there does not exist such reparametrization of the $F_{i}$ 's that

- would preserve the manifest symmetries,

- would produce the $\mathrm{F}_{5} \partial_{\mu} \mathrm{F}_{6} \partial^{\mu} \mathrm{F}_{7}$ term,

- would not change the polynomial structure of the other metric and torsion components.

The proof is completed by writing down the most general reparametrization, compatible with the first two requirements and by showing that the last requirement eliminates all potential free terms in it.

Therefore, at two loops, within the subspace spanned by $\lambda$ and $g$, the $\mathrm{F}_{1}$ dual of eq. (24) is not renormalizable in the ordinary, field theoretical sense, and the 
2 loop $\beta$-functions cannot be extracted. Thus, naively, one is tempted to conclude that the $F_{1}$ dual cannot be equivalent to the original model. However, since this non renormalizability follows from the appearance of new terms, not accounted for by reparametrizations, one has to understand this phenomenon better, before saying anything definit about the quantum equivalence between eq.(24) and $\mathcal{L}^{d}$. There are two points one has to take into account: the first - as we emphasized earlier - is that $\mathcal{L}^{d}$ has fewer symmetries than $\mathcal{L}$, eq. $(24)$, thus the appearance of new terms may be attributed to this symmetry reduction. The second point worth emphasizing is that describing $\mathcal{L}$ in terms of $\lambda$ and $g$ is not renorm invariant; for this one should use a quantity, $C=C(\lambda, g)$, depending on $\lambda$ and $g$, which is constant along the renorm trajectories: $\frac{\partial C}{\partial \lambda} \beta_{\lambda}(\lambda, g)+\frac{\partial C}{\partial g} \beta_{g}(\lambda, g)=0$, and the 'physical' $\beta$-function is obtained from $\beta_{\lambda}(\lambda, g)$ by expressing in it $g$ in terms of $C$. Therefore, putting these two observations together, it is conceivable, that for $\mathcal{L}^{d}$, working in a larger parameter space (i.e. having more parameters than just $\lambda, g$ ), and requireing the renorm invariants to be constants even at two loops forces us out of the $(\lambda, g)$ subspace, yet when the parameters are expressed in terms of the invariants, the physical $\beta^{d}$ still coincides with that of the original model.

To close this section we mention that we found qualitatively the same behaviour for the $\mathrm{F}_{2}$ dual of the simplest deformed principal sigma model. The global symmetries of this model contain not only $S L(3)_{L} \times \mathrm{R}^{2}$ but also the $\mathrm{Z}_{2}$ subgroup of $\mathrm{S}_{3}$ generated by $M_{1}$, eq.(18), as the action of $M_{1}$ on the fields, eq.(23), commutes with the translations of $\mathrm{F}_{2}$. Nevertheless, while in one loop we found the $\beta$-functions, eq.(31), at two loops new terms, similar to those in eq.(35), arose in the counterterms, and they destroyed the renormalizabilty of this model in the same way as in the case of the $\mathrm{F}_{1}$ dual.

\section{Models with more parameters}

\section{$\underline{5.1 \text { Derivation of the } \beta \text { functions }}$}

A systematic way to introduce more parameters into the Lagrangian, $\mathcal{L}^{d}$, of the previous section, is to construct it as the dual of the less symmetric deformed $S L(3)$ principal models described in sect.3. Of the many possibilities discussed there we choose the ' $M_{1}$ invariant', whose Lagrangian can be written as (see eq.19):

$$
\begin{aligned}
\mathcal{L} & =-\frac{1}{2 \lambda}\left(\operatorname{Tr} J_{\mu} J^{\mu}-4 b\left(J_{\mu}^{3}\right)^{2}-4 c\left(J_{\mu}^{8}\right)^{2}-d J_{\mu}^{1} J^{\mu 2}\right) \\
& =\frac{1}{2 \lambda} g_{i j}(\mathrm{~F}, b, c, d) \partial^{\mu} \mathrm{F}^{i} \partial_{\mu} \mathrm{F}^{j},
\end{aligned}
$$

since this is the only one where the counterterms reproduce the structure of the Lagrangian thus the two loop renormalization requires no reparametrization of the 
$\mathrm{F}_{\mathrm{i}}$ 's. The explicit form of the metric

$$
\begin{aligned}
& g_{11}=-2(1-2 b) \quad g_{16}=-2(1-2 b) \mathrm{F}_{3} \quad g_{17}=(1-2 b)\left(\mathrm{F}_{4}+\mathrm{F}_{3} \mathrm{~F}_{6} \mathrm{~F}_{4}+\mathrm{F}_{6} \mathrm{~F}_{5}\right) \\
& g_{18}=-(1-2 b)\left(\mathrm{F}_{3} \mathrm{~F}_{4}+\mathrm{F}_{5}\right) \quad g_{27}=-\sqrt{3}\left(\mathrm{~F}_{4}-\mathrm{F}_{6} \mathrm{~F}_{5}+\mathrm{F}_{3} \mathrm{~F}_{6} \mathrm{~F}_{4}\right)(1-2 c) \\
& g_{22}=4 c-2 \quad g_{28}=\sqrt{3}\left(-\mathrm{F}_{5}+\mathrm{F}_{3} \mathrm{~F}_{4}\right)(1-2 c) \\
& g_{36}=-1+\frac{d}{2} \quad g_{37}=-\frac{d}{2} \mathrm{~F}_{6} \mathrm{~F}_{4} \quad g_{38}=\frac{d}{2} \mathrm{~F}_{4} \quad g_{47}=-1-\mathrm{F}_{3} \mathrm{~F}_{6} \quad g_{58}=-1 \\
& g_{66}=(4 b-d) \mathrm{F}_{3}{ }^{2} \quad g_{67}=\left(\frac{d}{2}-2 b\right)\left(\mathrm{F}_{3}{ }^{2} \mathrm{~F}_{6} \mathrm{~F}_{4}+\mathrm{F}_{3} \mathrm{~F}_{6} \mathrm{~F}_{5}\right)+\frac{d}{2} \mathrm{~F}_{5}+(1-2 b) \mathrm{F}_{3} \mathrm{~F}_{4} \\
& g_{77}=-x\left(\mathrm{~F}_{5}{ }^{2} \mathrm{~F}_{6}{ }^{2}+\mathrm{F}_{3}{ }^{2} \mathrm{~F}_{6}{ }^{2} \mathrm{~F}_{4}{ }^{2}+2 \mathrm{~F}_{6} \mathrm{~F}_{4}{ }^{2} \mathrm{~F}_{3}+\mathrm{F}_{4}{ }^{2}\right)+y\left(\mathrm{~F}_{6} \mathrm{~F}_{4} \mathrm{~F}_{5}+\mathrm{F}_{3} \mathrm{~F}_{6}{ }^{2} \mathrm{~F}_{4} \mathrm{~F}_{5}\right) \\
& g_{48}=\mathrm{F}_{3} \quad g_{78}=x\left(\mathrm{~F}_{3}{ }^{2} \mathrm{~F}_{4}{ }^{2} \mathrm{~F}_{6}+\mathrm{F}_{3} \mathrm{~F}_{4}{ }^{2}+\mathrm{F}_{6} \mathrm{~F}_{5}{ }^{2}\right)-y\left(\frac{1}{2} \mathrm{~F}_{4} \mathrm{~F}_{5}+\mathrm{F}_{3} \mathrm{~F}_{6} \mathrm{~F}_{4} \mathrm{~F}_{5}\right) \\
& g_{57}=\mathrm{F}_{6} \quad g_{68}=\left(2 b-\frac{d}{2}\right)\left(\mathrm{F}_{4} \mathrm{~F}_{3}{ }^{2}+\mathrm{F}_{5} \mathrm{~F}_{3}\right) \quad g_{88}=x\left(\mathrm{~F}_{5}{ }^{2}+\mathrm{F}_{4}{ }^{2} \mathrm{~F}_{3}{ }^{2}\right)+y \mathrm{~F}_{5} \mathrm{~F}_{3} \mathrm{~F}_{4},
\end{aligned}
$$

where $x \equiv-b-3 c$ and $y \equiv 2 b-d-6 c$, reveals that the terms appearing in eq.(35) are already present in the original model. The formulae of the duality transformations, eq.(3), guarantee that these terms are also there in the Lagrangian of both the $F_{1}$ and the $\mathrm{F}_{2}$ duals of eq.(37). Setting $d \equiv 0$ and $b=c=-g / 2$ in eq.(37) yields the Lagrangian of the simplest deformed principal model, eq.(24).

We applied the coupling constant renormalization procedure for the 4 parameter purely metric sigma model, eq.(37), as well as for its $\mathrm{F}_{1}$ and $\mathrm{F}_{2}$ duals. In all cases it turned out that in this larger parameter space the $\Sigma_{1}$ and $\Sigma_{2}$ counterterms reproduce the structure of the corresponding Lagrangians, and up to two loops each of the three models proved to be renormalizable. The one loop $\beta$-functions

$$
\begin{aligned}
\beta_{\lambda}^{1} & =-\frac{\lambda^{2}(d+6 c+6+2 b)}{8 \pi}, \\
\beta_{b}^{1} & =\frac{\lambda\left(d^{2}+d+3 d c-b d-12 b c-12 b^{2}+8-4 b\right)}{16 \pi}+\frac{8 \lambda b(1-b)-2 \lambda}{2 \pi(d-2)^{2}} \\
& +\frac{\lambda(1-2 b)}{2 \pi(d-2)}, \\
\beta_{c}^{1} & =-\frac{\lambda\left(2 c d-d+36 c^{2}-2 b-18 c+4 b c\right)}{16 \pi}, \\
\beta_{d}^{1} & =\lambda\left(-\frac{d^{2}+3 c d+b d+4-6 c-2 b}{4 \pi}+\frac{4 b-2}{\pi(d-2)}\right),
\end{aligned}
$$

coincide in all three cases, while the case by case different, and somewhat more complicated two loop expressions are given in Appendix A. (In a form appropriately transformed to describe the evolution of the parameters introduced in eq.(42)). 
These $\beta$-functions contain some terms depending in a non polynomial way on the parameter $d$. This reflects our assumption that the $y_{K}(\lambda, b, c, d)$ residues of the simple poles in the coupling and wave function renormalization constants, $Z_{K}=$ $1+y_{k}(\lambda, b, c, d) / \epsilon+\ldots,(K=\overline{1,8}, \lambda, b, c, d)$ depend polynomially only on the coupling constant, $\lambda$, while they may depend in an arbitrary way on $b, c, d$, as in these parameters we do not expand anything. Note also that for $d \equiv 0$ and $b=c=-g / 2$ the expressions in eq.(39) become equivalent to the 'universal' 2 parameter one loop $\beta$-functions, eq.(31), while the metric 2 loop $\beta$-functions in Appendix A reduce to eq.(32).

Now that we succesfully obtained the $\beta$-functions up to two loops not only for the original model, eq.(37), but also for its two duals, and these $\beta$-functions are different from each other, we can discuss the quantum equivalence among them. To this end - as discussed at length in ref.[7] - first one should determine the renorm invariants $N(\lambda, b, c, d)$,

$$
\frac{\partial N}{\partial \lambda} \beta_{\lambda}+\frac{\partial N}{\partial b} \beta_{b}+\frac{\partial N}{\partial c} \beta_{c}+\frac{\partial N}{\partial d} \beta_{d}=0
$$

characterizing the trajectories for each model in question. Eq.(40) is a homogeneous, linear partial differential equation, thus by general theorems it admits - at least locally - , in the present case, 3 independent first integrals $N_{i}(\lambda, b, c, d) i=1,2,3$. Then, for each model, one should solve (of course only perturbatively in $\lambda$ ) the $N_{i}(\lambda, b, c, d)=$ $N_{i}^{0}$ equations for $b, c, d$ and use the $b=b\left(\lambda, N_{i}^{0}\right)$ etc. expressions in the corresponding $\beta_{\lambda}$ to obtain the 'physical' $\beta$-functions. Then the models may be equivalent if the various physical $\beta$-functions coincide for the same values of $N_{i}^{0}$.

The actual integration of eq.(40) is a formidable task that greatly exceeds our capabilities. This is so even if we realise that the first step would be to determine $N_{i}(\lambda, b, c, d)$ for the one loop $\beta$-functions, eq.(39), common for all three models, and the two loop corrections could be obtained perturbatively. What we can do is a sort of 'fixed point analysis': we linearize (in $b, c, d$ ) the various $\beta$-functions around the particular $b, c, d$ values ('fixed points'), satisfying

$$
\beta_{b}=\beta_{c}=\beta_{d}=0
$$

and use them in eq.(40).

Though the one loop $\beta$-functions, eq.(39) allow a number of real fixed points

$$
\begin{aligned}
& (b=c=d=0), \quad(b=c=1 / 2 d=0), \quad(b=c=1 / 2 d=-2), \\
& (b=-1 / 2 c=1 / 2 d=-2), \quad(b=3 / 10 c=1 / 2 d=6 / 5),
\end{aligned}
$$


it is only the $(b=c=1 / 2 d=0)$ one that makes all three two loop sets of $\beta_{b}, \beta_{c}$, and $\beta_{d}$ vanish. Therefore we carry out the the aforementioned analysis for this 'fixed point', which, incidentally, describes the coset model where the $J_{\mu}^{3}$ and $J_{\mu}^{8}$ current components decouple. The analysis becomes tractable by introducing the $B, C, D$ parameters:

$$
b=-\frac{1}{2}(B(D+1)+D), \quad c=-\frac{1}{2} C-\frac{1}{6} D, \quad d=-2 D,
$$

in terms of which the one loop $\beta$-functions simplify:

$$
\begin{aligned}
& \beta_{\lambda}^{(1)}=\frac{\lambda^{2}(B+B D+4 D-6+3 C)}{8 \pi}, \quad \beta_{B}^{(1)}=\frac{B \lambda\left(5+2 D+D^{2}\right)(B+1)}{4 \pi(1+D)}, \\
& \beta_{C}^{(1)}=\frac{\lambda\left(6 B+2 B D+3 B D C+3 B C+24 C+27 C^{2}+24 D C+16 D\right)}{24 \pi}, \\
& \beta_{D}^{(1)}=\frac{\lambda\left(B D^{2}+6 D^{2}+2 D-3 B+2 B D+3 C+3 D C\right)}{8 \pi},
\end{aligned}
$$

and also the two loop expressions acquire a somewhat less complicated form. In terms of these new parameters $(B=-1, C=-1, D=0)$ is the fixed point describing the coset model, while the subspace corresponding to the most symmetric deformed principal model (and its duals) is obtained by the substitution $B \equiv C \equiv g, D \equiv 0$.

$\underline{5.2 \text { Analysis around the 'fixed point' }}$

We linearize the three one and two loop $\beta$ functions $\beta_{B}, \beta_{C}, \beta_{D}$, by writing

$$
B=-1+\hat{b}, \quad C=-1+\hat{c}, \quad D=0+\hat{d},
$$

for both the metric model, eq.(37), as well as for the $\mathrm{F}_{1}$ and $\mathrm{F}_{2}$ duals. (We emphasize, that the linearization does not effect the $\lambda$ dependence). Assembling $[\hat{b}, \hat{c}, \hat{d}]$ into the components of a vector, the right hand sides of eq.(43) and (A.2-A.4), (A.6-A.8), (A.10-A.12), yield in each case a $3 \times 3$ matrix acting on $[\hat{b}, \hat{c}, \hat{d}]$, whose eigenvalues and eigenvectors play an important role in the following. For the metric model the eigenvalues and the corresponding eigenvectors of this matrix are:

$$
\begin{array}{ll}
-\frac{\lambda}{16 \pi^{2}}(20 \pi+23 \lambda) & {\left[1,0, \frac{3}{7}+\frac{345}{196 \pi} \lambda\right]} \\
-\frac{\lambda}{16 \pi^{2}}(20 \pi+23 \lambda) & {\left[0,1,-\frac{3}{7}-\frac{345}{196 \pi} \lambda\right]} \\
-\frac{\lambda}{16 \pi^{2}}(8 \pi+31 \lambda) & {[0,1,-3]}
\end{array}
$$


while for the the $\mathrm{F}_{1}$ and $\mathrm{F}_{2}$ duals the results coincide:

$$
\begin{array}{ll}
-\frac{\lambda}{16 \pi^{2}}(20 \pi+23 \lambda) & {\left[1-\frac{2 \lambda}{\pi}, 1,0\right],} \\
-\frac{\lambda}{16 \pi^{2}}(20 \pi+23 \lambda) & {\left[\frac{7}{3}-\frac{41}{4 \pi} \lambda, 0,1\right]} \\
-\frac{\lambda}{16 \pi^{2}}(8 \pi+31 \lambda) & {[0,1,-3] .}
\end{array}
$$

In these expressions the terms linear (constant) in $\lambda$ represent the one loop results, while the quadratic (linear) ones describe the two loop corrections. Note that up to this order the three eigenvalues for the metric and the $F_{1}, F_{2}$ duals are the same in spite of the different $\beta$ functions. However the two loop eigenvectors in case of the $\mathrm{F}_{1} / \mathrm{F}_{2}$ duals are different from the metric ones.

Keeping only the constant and linear terms of $\hat{b}, \hat{c}$, and $\hat{d}$ also in $\beta_{\lambda}$ simplifies the one loop renormalization equations, eq.(43):

$$
\frac{d \lambda}{d t}=-\frac{\lambda^{2}}{8 \pi}\left(\sum_{j} a_{j} v_{j}+L\right) ; \quad \frac{d v_{i}}{d t}=\frac{\lambda}{8 \pi} \Delta_{i} v_{i}
$$

where $\frac{\lambda}{8 \pi} \Delta_{i}(i=1,2,3)$ denote the three (1 loop) eigenvalues appearing in eq. $(45,46)$, $v_{i}$ stand for the components of the corresponding eigenvectors, and in the last equation there is no summation over $i$. These equations admit the following renorm invariants:

$$
N_{i}=\frac{\lambda}{v_{i}^{-L / \Delta_{i}}} \exp \left(\sum \frac{a_{k}}{\Delta_{k}} v_{k}\right), \quad i=1,2,3 .
$$

Nevertheless this is not yet the end of the story. The point is that expressing $v_{i}=$ $v_{i}\left(\lambda, N_{j}\right)$ from eq.(48) makes sense in perturbation theory only if $v_{i}$ stay small for $\lambda \rightarrow 0$; i.e. if

$$
0<-\frac{L}{\Delta_{i}} \leq 1
$$

Therefore our analysis yields acceptable results only if these conditions are satisfied; it is easy to see that eq. (49) holds for the two coinciding eigenvalues in eq. $(45,46)$, (as $L=10$ and $\left.\Delta_{1,2}=-10\right)$, while it is not satisfied for the third eigenvalue. Therefore our perturbative results are valid only in the $\left[\hat{b}, \hat{c}, \frac{3}{7}(\hat{b}-\hat{c})\right]\left(\left[\hat{c}+\frac{7}{3} \hat{d}, \hat{c}, \hat{d}\right]\right)$ subspaces of the three dimensional space spanned by $\hat{b}, \hat{c}, \hat{d}$ for the metric and the $\mathrm{F}_{1} / \mathrm{F}_{2}$ dual models respectively.

The fact that according to eq. $(45,46)$ these "perturbative" subspaces change little (to $\left[\hat{c}+\frac{7}{3} \hat{d}-\frac{\lambda}{\pi}\left(2 \hat{c}+\frac{41}{4} \hat{d}\right), \hat{c}, \hat{d}\right]$ for the $\mathrm{F}_{1} / \mathrm{F}_{2}$ dual) or none $\left(\left[\hat{b}, \hat{c}, \frac{3}{7}(\hat{b}-\hat{c})\right]\right.$ stays for the 
metric model) in the two loop order already indicates, that they are indeed appropriate to describe the perturbative evolution of these models. To prove this more rigorously in Appendix B we show that in these subspaces the two loop corrections to $N_{i}$ are indeed of higher order in $\lambda$.

In the perturbative subspaces - exploiting the degeneracy of the corresponding eigenvalues - the $\lambda$ dependence of the remaining two parameters becomes $p_{i} \sim \frac{\lambda}{N_{i}}+$ $o\left(\lambda^{2}\right)$, where $p_{1}=\hat{c}, p_{2}=\hat{d}$ as they effectively arise from the one loop results. Since the two loop $\left(o\left(\lambda^{3}\right)\right)$ corrections to $\beta_{\lambda}$ come in the form $\lambda^{3} \otimes(B=C=-1, D=$ 0 )fixed point $+\lambda^{2} \otimes o(\lambda)$ corrections of the one loop terms; and the one loop terms are common for all three models, the "physical" $\beta_{\lambda}$-s of the various models coincide if the $\lambda^{3} \otimes$ fixed point pieces coincide. Looking at the explicit expressions in Appendix A reveals that this is indeed the case; i.e. in the perturbative subspaces, up to two loops, the three models may indeed be equivalent.

The two loop form of the perturbative subspace of the $\mathrm{F}_{1} / \mathrm{F}_{2}$ duals, $\left[\hat{c}+\frac{7}{3} \hat{d}-\right.$ $\left.\frac{\lambda}{\pi}\left(2 \hat{c}+\frac{41}{4} \hat{d}\right), \hat{c}, \hat{d}\right]$, as compared to the metric one $\left[\hat{b}, \hat{c}, \frac{3}{7}(\hat{b}-\hat{c})\right]$, also explaines why we needed to enlarge the parameter space of the simplest deformed principal model, which corresponds to $\hat{b} \equiv \hat{c}, \hat{d} \equiv 0$ : while this is a consistent subspace of the metric two loop perturbative subspace it is not consistent with that of the $F_{1} / F_{2}$ duals. Indeed, for the latter case, even if we put $\hat{d}=0$ in the one loop order, to have $\hat{b}=\hat{c}$ in two loops we need a non vanishing $\hat{d}$ of $o(\lambda)$. The interpretation of this is clear: since the dual models have less symmetry than the simplest deformed principal model there is nothing that would prohibit the perturbative generation of the coupling described by $\hat{d}$. Nevertheless - at least when we treat the deviation of $g \equiv B \equiv C$ from $-1, g=\hat{c}-1$, perturbatively, - our previous findings about the coinciding physical $\beta$ functions for the metric and the $\mathrm{F}_{1} / \mathrm{F}_{2}$ dual models indicate, that the simplest deformed principal model and its $\mathrm{F}_{1} / \mathrm{F}_{2}$ duals in the extended parameter space may, indeed, be physically equivalent.

\section{Summary and conclusions}

In this paper we investigated the quantum equivalence between various deformed $S L(3)$ principal models and their different duals in the two loop order of perturbation theory. The investigation is based on extracting and comparing various $\beta$ functions of the original and dual models.

To keep the number of parameters that require renormalization small, as a first step, we determined the Lagrangians of those deformed $S L(3)$ principal models that admit $S L(3)_{L} \times R^{2} \times$ some discrete subgroup of $S L(3)$ as global symmetries. Then 
we carried out the coupling constant renormalization program and determined the various $\beta$ functions for the simplest (i.e. most symmetric) deformed $S L(3)$ principal model as well as for the more general case with $S L(3)_{L} \times R^{2} \times Z_{2}$ symmetry.

We considered only those duals of these sigma models (we called them $\mathrm{F}_{1}$ and $\mathrm{F}_{2}$ duals) that are obtained by a very simple Abelian duality transformation when the $g_{00}$ component of the metric is constant. This choice is motivated - besides beeing obviously the simplest possibity - also by the following: the question of quantum equivalence between the original and dual models is basically a two loop problem, as up to one loop the equivalence is shown in general in ref.[8]. However to determine the two loop counterterms of the duals obtained by using a non constant $g_{00}$ exceeded greatly the power of our computers. The case of constant $g_{00}$ is also favoured by the fact, that in this case the standard (functional integral based) derivation of the duality transformations amounts to a standard Gaussian integration, thus no problems are expected with the quantum equivalence of the two models.

Of the counterterms of the $\mathrm{F}_{1}$ and $\mathrm{F}_{2}$ duals of the simplest deformed $S L(3)$ principal model we found that in the two loop order new terms appeared that could not be accounted for by some field redefinition. Therefore, naively, these duals seem to be non renormalizable in the ordinary, field theoretic sense. However, since for these dual models some of the explicit discrete symmetries of the simplest deformed $S L(3)$ principal model are absent, we conjectured, that the appearance of the new terms may be the consequence of this symmetry reduction. Thus working in a larger parameter space the renormalizability of the dual models would be restored and expressing everything in terms of renorm invariants, the original and dual $\beta$ functions could still coincide.

We verified explicitly, without any approximation, the restoration of the renormalization of the dual models in an appropriately chosen larger parameter space. However we could verify the equality of the various 'physical' $\beta$ functions only by treating the deviations of these parameters from certain 'fixed point values' perturbatively. Nevertheless these are certainly consistent with the expectation that - in case of a constant $g_{00}$ - the equivalence of the dually related sigma models also involves a change of the renormalization scheme.

We close the paper by an additional - not entirely unrelated - speculation about the origin of the new terms appearing in the two loop counterterms of the $\mathrm{F}_{1}$ and $\mathrm{F}_{2}$ duals of the simplest deformed $S L(3)$ principal model. This speculation is based on a recent proposal [15] according to which the naive duality transformation rules for the renormalized quantities (as opposed to the bare ones) receive perturbative 
corrections beyond one loop. These corrections are encoded into a mapping $\gamma_{i j}(g, b)=$ $g_{i j}+b_{i j}+\alpha^{\prime} M_{i j}(g, b)+\ldots$ that solves the following equation:

$$
\tilde{T}^{(0)}(g, b)=\left(\gamma^{-1} \circ T^{(0)} \circ \gamma\right)(\tilde{g}, \tilde{b}),
$$

where $T^{(0)}(g, b)$ stands for the generalized bare metric, that can be expressed in terms of the renormalized quantities as:

$$
T_{i j}^{(0)}(g, b)=g_{i j}+b_{i j}+\frac{\alpha^{\prime}}{\epsilon} \hat{R}_{i j}(g, b)+\frac{\left(\alpha^{\prime}\right)^{2}}{\epsilon} \hat{Y}_{i j}(g, b)+\ldots,
$$

$\left(\hat{Y}_{i j}=\frac{1}{8} Y_{j}^{l m k} \hat{R}_{i k l m},\right)$ and $\sim$ denotes the naive dual, given by eq.(3), for both $g, b$ and also for $T^{(0)}$. In ref.[15] eq.(50) was solved for the special, block diagonal metric case, when in the adapted coordinate system $b_{i j} \equiv 0 g_{0 \alpha} \equiv 0$ and $g_{00}=g_{00}\left(\xi^{\alpha}\right)$. The examples of the $F_{1}$ and $F_{2}$ duals we consider here belong to another special case when $g_{00}$ is constant but $g_{0 \alpha} \neq 0$. Thus we conjecture that the new terms that appear are the manifestations of the presence of the $\gamma$ mapping, whose general form is not known yet. We hope to return to the discussion of this possibility elsewhere.

Acknowledgements This research was supported in part by the Hungarian National Science and Research Fund (OTKA) T016251.

\section{Appendix A}

In this appendix we describe the explicit form of the two loop $\beta$ functions for the various parameters introduced in eq.(42). For the metric model, eq.(37), they take the form:

$$
\begin{gathered}
\beta_{\lambda}=-\frac{\lambda^{2}}{32 \pi^{2}}(-12 \pi C+24 \pi-4 \pi B D-16 \pi D-4 \pi B) \\
-\frac{\lambda^{3}}{32 \pi^{2}}\left(5 B^{2}+3 B D C+18 C^{2}-9 D-2 B+9+3 D B+21 D C\right. \\
\left.+11 D^{2}+5 B D^{2}+3 B C-9 C+4 B^{2} D+2 B^{2} D^{2}\right) \\
\beta_{B}=B(1+B)\left(\frac{\lambda}{64(1+D)^{2} \pi^{2}}\left(48 \pi D^{2}+112 \pi D+16 \pi D^{3}+80 \pi\right)\right. \\
+\frac{\lambda^{2}}{64(1+D)^{2} \pi^{2}}\left(-20 D B-43 B+4 B D^{3}+B D^{4}-6 B D^{2}+52+30 D+9 D C\right. \\
\left.\left.+6 D^{2}+3 C+6 D^{4}+3 D^{3} C+2 D^{3}+9 D^{2} C\right)\right) \\
\beta_{C}=-\frac{\lambda}{192 \pi^{2}}\left(-192 \pi C-216 \pi C^{2}-16 \pi B D-128 \pi D-48 \pi B-192 \pi D C-24 B \pi C-24 B D C \pi\right) \\
\left(-\frac{1}{192 \pi^{2}}\left(-8-104 D_{-6} D B-90 D C+22 B D^{2}-18 B C+28 B^{2} D+8 B^{2} D^{2}+9 C^{2} D B\right.\right. \\
-114 C-26 B+32 B^{2}-90 C^{2}+16 D^{2}+24 B D^{2} C+24 B^{2} D C+6 B D C+12 B^{2} D^{2} C+4 D^{3} \\
\left.+81 C^{3}+2 B D^{3}+42 D^{2} C+9 C^{2} B+72 C^{2} D+30 B^{2} C\right) \\
\left.-\frac{1+5 B^{2}-2 B}{24 \pi^{2}(1+D)}\right) \lambda^{2}
\end{gathered}
$$




$$
\begin{aligned}
& \beta_{D}=-\frac{\lambda}{64 \pi^{2}}\left(-24 \pi D C-48 \pi D^{2}-16 \pi B D-16 \pi D-24 \pi C-8 \pi B D^{2}+24 \pi B\right) \\
& \quad\left(-\frac{1}{64 \pi^{2}}\left(-2 B^{2}+18 D C+6 B C+6 B^{2} D+12 B^{2} D^{2}+7 B D^{2}\right.\right. \\
& +36 C^{2}-21 C+12 B D C+5 B+8-4 D+5 D B+6 B D^{2} C+39 D^{2} C \\
& \left.+7 B D^{3}+4 B^{2} D^{3}+14 D^{3}+36 C^{2} D+2 D^{2}\right) \\
& \left.\quad+\frac{1+5 B^{2}-2 B}{8 \pi^{2}(1+D)}\right) \lambda^{2} .
\end{aligned}
$$

For the $\mathrm{F}_{1}$ dual the corresponding expressions are

$$
\begin{gathered}
\beta_{\lambda}=-\frac{\lambda^{3}}{32 \pi^{2}}\left(8-6 B+18 C^{2}+D^{2} B^{2}+3 D^{2} B+2 D B^{2}+21 C D+3 B D C\right. \\
\left.+2 B^{2}+10 D^{2}+3 B C-5 D-9 C+5 D B\right) \\
-\frac{\lambda^{2}}{16 \pi^{2}}(12 \pi-2 \pi B-8 \pi D-2 \pi B D-6 \pi C), \\
\beta_{B}=-\frac{\lambda^{2}}{64 \pi^{2}(1+D)^{2}}(1+B)\left(12 D^{3} B^{2}-2 B D^{4}+22 D^{2} B^{2}+20 D B^{2}+55 B^{2}-3 B C+24 B\right. \\
\left.+2 D B+26 D^{2} B+14 D^{3} B-3 C B D^{3}-9 D^{2} B C-9 B D C+3 D^{4} B^{2}+64+16 D^{2}+32 D\right) \\
-\frac{\lambda}{32 \pi^{2}(1+D)^{2}}(1+B)\left(-8 \pi D^{3} B-56 \pi B D-24 \pi D^{2} B-40 \pi B\right), \\
\beta_{C}=-\frac{\lambda^{2}}{192 \pi^{2}(1+D)}\left(60+12 D-54 C^{2}+36 B^{2}+4 D^{3} B^{2}-18 B-48 C+4 D^{2}-18 C D+16 D^{2} B^{2}\right. \\
+88 D^{2} B+24 D B^{2}+30 B C+144 B D C+56 D B+90 B C^{2} D+2 B D^{4}+32 D^{3} B+36 C B D^{3} \\
+150 D^{2} B C+4 D^{4}+32 D^{3}+81 C^{3}+45 B D^{2} C^{2}+6 C D^{3} B^{2}+18 C D^{2} B^{2} \\
\left.+24 C D B^{2}+108 D^{2} C^{2}+90 D^{2} C+60 C D^{3}+54 C^{2} D+12 C B^{2}+45 B C^{2}+81 D C^{3}\right) \\
-\frac{\lambda}{96 \pi^{2}(1+D)}\left(-12 B \pi C-24 \pi B-64 \pi D-96 \pi C-24 B \pi C D-12 B D^{2} \pi C\right. \\
\left.-192 \pi C D-64 \pi D^{2}-108 \pi C^{2}-8 \pi D^{2} B-32 \pi B D-96 D^{2} \pi C-108 D \pi C^{2}\right), \\
\beta_{D}=-\frac{\lambda^{2}}{64 \pi^{2}(1+D)}\left(-30-8 D+36 C^{2}-24 B^{2}+8 D^{3} B^{2}+9 B-21 C+18 D^{2}-3 C D\right. \\
+10 D^{2} B^{2}+24 D^{2} B+4 D B^{2}+6 B C+18 B D C-2 D B+2 D^{4} B^{2}+7 B D^{4}+26 D^{3} B \\
\left.+6 C B D^{3}+18 D^{2} B C+16 D^{4}+36 D^{3}+36 D^{2} C^{2}+57 D^{2} C+39 C D^{3}+72 C^{2} D\right) \\
-\frac{\lambda}{32 \pi^{2}(1+D)}\left(4 \pi B D+12 \pi B-8 \pi D-12 \pi C-12 D^{2} \pi C\right. \\
\left.-24 \pi C D-32 \pi D^{2}-12 \pi D^{2} B-24 \pi D^{3}-4 \pi D^{3} B\right),
\end{gathered}
$$

while for the $\mathrm{F}_{2}$ dual they are:

$$
\begin{aligned}
& \beta_{\lambda}=\left(-\left(10+2 B^{2}+5 B\right) D^{2}-\left(4 B^{2}+3 C B-9+15 C+3 B\right) D\right. \\
& \left.\quad-\left(18-2 B-9 C+5 B^{2}+3 C B+9 C^{2}\right)\right) \frac{\lambda^{3}}{32 \pi^{2}} \\
& \quad+((2 \pi B+8 \pi) D-(12 \pi-6 \pi C-2 \pi B)) \frac{\lambda^{2}}{16 \pi^{2}}
\end{aligned}
$$




$$
\begin{aligned}
\beta_{B}= & \left(\frac{B}{64 \pi^{2}}\left(2 D^{2}+3 B D^{2}-9 C D+B^{2} D^{2}+2 B^{2} D-26 D-9 C B D-24 B D-9 C-3 B-9 C B+8-11 B^{2}\right)\right. \\
+ & \left.\frac{B\left(1-B^{2}\right)}{2 \pi^{2}(D+1)^{2}}\right) \lambda^{2}+\left(\frac{B(8 \pi B D+8 \pi+8 \pi D+8 \pi B)}{32 \pi^{2}}+\frac{B(1+B)}{\pi(D+1)}\right) \lambda \\
\beta_{C}= & \left(-\frac{1}{192 \pi^{2}}\left(124-24 D-26 B+252 C+32 B^{2}+198 C^{2}-18 C B+28 D^{2}+30 C B^{2}\right.\right. \\
& +4 D^{3}+135 C^{3}+60 C D^{2}+144 C^{2} D+2 B D^{3}+9 C^{2} B+24 C B D^{2}+9 C^{2} B D+12 C B^{2} D^{2} \\
& \left.+24 C B^{2} D+22 B D^{2}+8 B^{2} D^{2}+78 C D+6 C B D-6 B D+28 B^{2} D\right) \\
& \left.-\frac{5 B^{2}-2 B+1}{24 \pi^{2}(D+1)}\right) \lambda^{2} \\
& -\frac{\lambda}{96 \pi^{2}}\left(-24 \pi B-12 C \pi B-96 \pi C-8 \pi B D-12 C \pi B D-96 \pi C D-108 \pi C^{2}-64 \pi D\right), \\
\beta_{D}= & \left(-\frac{1}{64 \pi^{2}}\left(-2 B^{2}+18 C^{2}+42 D+6 C B+38+7 B D^{2}+6 B^{2} D+12 B^{2} D^{2}+5 B+30 C D-9 C+12 C B D\right.\right. \\
+ & \left.\left.16 D^{3}+20 D^{2}+39 C D^{2}+18 C^{2} D+7 B D^{3}+6 C B D^{2}+4 B^{2} D^{3}+5 B D\right)+\frac{5 B^{2}-2 B+1}{8 \pi^{2}(D+1)}\right) \lambda^{2} \\
- & \frac{\lambda}{32 \pi^{2}}\left(-4 \pi B D^{2}-12 \pi C-8 \pi B D+12 \pi B-12 \pi C D-24 \pi D^{2}-8 \pi D\right) .
\end{aligned}
$$

\section{Appendix B}

In this appendix we show on the example of the perturbative subspace of the $\mathrm{F}_{1} / \mathrm{F}_{2}$ duals

$$
\hat{b}=\hat{c}+\frac{7}{3} \hat{d}-\frac{\lambda}{\pi}\left(2 \hat{c}+\frac{41}{4} \hat{d}\right)
$$

that the two loop corrections to $N_{i}$ are indeed of higher order in $\lambda$. On the surface defined by (B.1) in the space of $\hat{b}, \hat{c}, \hat{d}$ the appropriately linearized renormalization equations have the following form:

$$
\begin{aligned}
\frac{d \lambda}{d t} & =-\frac{\lambda^{2}}{8 \pi}\left(\alpha_{0} c+\beta_{0} d+L\right)-\frac{\lambda^{3}}{8 \pi^{2}}\left(\alpha_{1} c+\beta_{1} d+S\right), \\
\frac{d c}{d t} & =\frac{\lambda}{8 \pi} \Delta_{1} c-\frac{\lambda^{2}}{8 \pi^{2}} \tilde{\Delta}_{1} c, \\
\frac{d d}{d t} & =\frac{\lambda}{8 \pi} \Delta_{2} d-\frac{\lambda^{2}}{8 \pi^{2}} \tilde{\Delta}_{2} d,
\end{aligned}
$$

where all terms of $o\left(c^{2}, c d, d^{2}\right)$ are neglected and the various constants (whose actual values are not important for the rest) can be obtained from eq.(46) and (A.5-A.12). One verifies by direct computation that the expressions:

$$
\begin{gathered}
\tilde{N}_{1}=\frac{\lambda}{c^{-L / \Delta_{1}}} \exp \left(\frac{\alpha_{0}}{\Delta_{1}} c+\frac{\beta_{0}}{\Delta_{2}} d\right)\left(1+\lambda\left(P+k_{1} c+k_{2} d\right)\right), \\
\tilde{N}_{2}=\frac{\lambda}{d^{-L / \Delta_{2}}} \exp \left(\frac{\alpha_{0}}{\Delta_{1}} c+\frac{\beta_{0}}{\Delta_{2}} d\right)\left(1+\lambda\left(Q+l_{1} c+l_{2} d\right)\right),
\end{gathered}
$$


where

$$
\begin{aligned}
& P=-\frac{1}{\pi}\left(S+L \frac{\tilde{\Delta}_{1}}{\Delta_{1}}\right), \quad k_{1}=\frac{1}{\pi\left(\Delta_{1}-L\right)}\left(\alpha_{0} \pi P+\alpha_{1}+\alpha_{0} \frac{\tilde{\Delta}_{1}}{\Delta_{1}}\right), \\
& k_{2}=\frac{1}{\pi\left(\Delta_{2}-L\right)}\left(\beta_{0} \pi P+\beta_{1}+\beta_{0} \frac{\tilde{\Delta}_{2}}{\Delta_{2}}\right),
\end{aligned}
$$

and

$$
\begin{aligned}
& Q=-\frac{1}{\pi}\left(S+L \frac{\tilde{\Delta}_{2}}{\Delta_{2}}\right), \quad k_{1}=\frac{1}{\pi\left(\Delta_{1}-L\right)}\left(\alpha_{0} \pi Q+\alpha_{1}+\alpha_{0} \frac{\tilde{\Delta}_{1}}{\Delta_{1}}\right), \\
& k_{2}=\frac{1}{\pi\left(\Delta_{2}-L\right)}\left(\beta_{0} \pi Q+\beta_{1}+\beta_{0} \frac{\tilde{\Delta}_{2}}{\Delta_{2}}\right),
\end{aligned}
$$

are indeed invariants of the flow described by (B.2), provided we neglect $c^{2}, c d$, and $d^{2}$. From (B.3) one obtaines $p_{i} \sim \frac{\lambda}{\tilde{N}_{i}}+o\left(\lambda^{2}\right)$, with $p_{1}=\hat{c}, p_{2}=\hat{d}$. The $\tilde{N}_{i}$ and the $\lambda \rightarrow 0$ behaviour of the parameters in the perturbative subspace of the metric model are derived in an entirely analogous way.

\section{References}

[1] T. Buscher, Phys. Lett. B194 (1987) 51.

[2] E. Alvarez, L. Alvarez-Gaumé and Y. Lozano, Nucl. Phys. B (Proc. Suppl.) 41 (1995) 1; (hep-th/9410237).

[3] X. De la Ossa and E. Quevedo, Nucl. Phys. B403 (1993) 377.

E. Alvarez, L. Alvarez-Gaumé and Y. Lozano, Nucl. Phys. B424 (1994) 155.

[4] E. Alvarez, L. Alvarez-Gaumé and Y. Lozano, Phys. Lett. B336 (1994) 183.

[5] T. Curtright and C. Zachos, Phys. Rev. D49 (1994) 5408, and hep-th/9407044. Y. Lozano Phys. Lett. B355 (1995) 165.

[6] E. Fradkin and A.A. Tseytlin, Ann. Phys. 162 (1985) 31.

[7] J. Balog, P. Forgács, Z. Horváth, L. Palla, hep-th/9601091 Nucl. Phys. B (Proc. Suppl.)49 (1996) 16.

[8] P.E. Haagensen, hep-th/9604136

[9] C.M. Hull and P.K. Townsend, Phys. Lett. B191 (1987) 115.

[10] R.R. Metsaev and A.A. Tseytlin, Phys. Lett. B191 (1987) 354.

[11] H. Osborn, Ann. Phys. 200 (1990) 1.

[12] P.S. Howe, G. Papadopoulos and K.S. Stelle, Nucl. Phys. B296 (1988) 26. H. Osborn, Nucl. Phys. B294 (1987) 595.

[13] J. Balog, P. Forgács, Z. Horváth, L. Palla, unpublished.

[14] D. Friedan, Phys. Rev. Lett 45 (1980) 1057, Ann. Phys. 163 (1985) 318.

[15] J. Balog, P. Forgács, Z. Horváth, L. Palla, hep-th/9606187, to appear in Phys. Lett. B. 